\title{
Rates of Depression in Individuals With Pathologic But Not Clinical Alzheimer Disease are Lower Than Those in Individuals Without the Disease: Findings From the Baltimore Longitudinal Study on Aging (BLSA)
}

\author{
Melissa D. Morgan, BS,* Michelle M. Mielke, PhD,* Richard O'Brien, MD,* \\ Juan C. Troncoso, MD, ${ }^{*}$ Alan B. Zonderman, PhD, $\dagger$ and Constantine G. Lyketsos, MD, MHS*
}

\begin{abstract}
The prevalence of major depression is increased in Alzheimer disease (AD), but currently the basis of this association remains unclear. The present study examined rates of depression in 4 groups of participants with postmortem examination from the Baltimore Longitudinal Study of Aging: (1) cognitively normal controls with no Alzheimer pathology, (2) cognitively normal individuals with Alzheimer pathology, (3) individuals with mild cognitive impairment plus Alzheimer pathology, (4) individuals with clinical diagnoses of dementia plus Alzheimer pathology. Depressive symptoms were assessed using the Center for Epidemiologic Studies Depression Scale. Individuals with Alzheimer pathology but no cognitive decline before death had significantly lower rates of depression than cognitively normal controls with no Alzheimer pathology and individuals with Alzheimer pathology plus clinical diagnoses of dementia. These findings suggest that depression is a risk factor for $\mathrm{AD}$ in the presence of $\mathrm{AD}$ pathology, but depression is not a risk factor for AD pathology.
\end{abstract}

Key Words: Alzheimer disease, dementia, depression, autopsy

(Alzheimer Dis Assoc Disord 2007;21:199-204)

A lzheimer disease (AD) is a devastating neurodegenerative disease and the most common cause of dementia. Over 4.5 million people have $\mathrm{AD}$, a number that is expected to triple as the population ages, ${ }^{1}$ creating a major public health burden. Furthermore, AD is a psychiatric disease, as $90 \%$ of $\mathrm{AD}$ patients also have noncognitive psychiatric symptoms such as agitation,

Received for publication October 18, 2006; accepted June 18, 2007.

From the *Johns Hopkins University; and $\uparrow$ National Institute on Aging, Baltimore, MD

Supported in part by the William C. Walker Scholarship, the Johns Hopkins University School of Medicine Dean's Fund, the Johns Hopkins Alzheimer Disease Research Center (5 PO1 AGO5146), and by the National Institute on Aging Intramural Research Program of the National Institutes of Health.

Reprints: Constantine G. Lyketsos, MD, MHS, Department of Psychiatry Johns Hopkins Bayview, 4940 Eastern Avenue, A4 Center, Room 458, Baltimore, MD 21224 (e-mail: kostas@jhmi.edu).

Copyright $(\odot 2007$ by Lippincott Williams \& Wilkins delusions, and hallucinations. ${ }^{2}$ Major depression, with a prevalence rate of $24 \%$, is one of the most common psychiatric syndromes observed in $\mathrm{AD} .{ }^{3}$ Because this is a much higher prevalence than the rate found in a comparable population without dementia $(7 \%),{ }^{3}$ numerous studies have investigated the basis of the association between AD and depression.

Currently, it is unclear whether depression represents a risk factor for AD or is an early clinical state of the disorder, as research has supported both hypotheses. The relative risk of developing AD increases with increasing number of depressive symptoms. ${ }^{4}$ Dal Forno et $\mathrm{al}^{5}$ found that symptoms of depression were a risk factor for AD in men, but not women, and concluded that depressive symptomatology is a risk factor for, but not a prodrome of dementia. In another study, the age of onset of $\mathrm{AD}$ was, on average, 25 years later than the onset of major depressive disorder, again suggesting that major depressive disorder may be a risk factor rather than a prodrome. ${ }^{6}$ Alternatively, some prospective studies have found that depressive symptoms are increased in preclinical $\mathrm{AD}$, and that they are not simply a response to self-perceived cognitive problems. For example, Berger et $\mathrm{al}^{7}$ found increased depressive symptoms 3 years before the diagnosis of $\mathrm{AD}$, and Geerlings et $\mathrm{al}^{8}$ suggest that depression may be an early symptom or subclinical expression of dementia in more highly educated elderly. Furthermore, Gatz et $\mathrm{al}^{9}$ found no significant association between a past history of depression and risk of developing $\mathrm{AD}$, but did find that people with clinical depression at the time of visit were over twice as likely to have AD 5 years later, suggesting depression may in fact be a preclinical state of AD.

Current research is also focused on identifying the pathophysiologic basis of depression in AD. One hypothesis is that the neuritic plaques and neurofibrillary tangles (NFT) that characterize AD pathology also underlie the depression associated with dementia. In a preliminary study, Sweet et al ${ }^{10}$ examined a small number of subjects with late life major depression (LLMD) who developed dementia and found that $86 \%$ had AD pathology on autopsy. However, all of the subjects with AD pathology and LLMD also had comorbid 
cerebrovascular disease or dementia with Lewy bodies pathology, indicating that AD pathology is probably not the sole contributor to LLMD.

Other studies have reported different results. Wilson et $\mathrm{al}^{11}$ concluded that the association between depression and $\mathrm{AD}$ is independent of $\mathrm{AD}$ pathology, as neither regionality nor severity of pathology were significantly related to depression scores. Thomas et al ${ }^{12}$ compared individuals with a history of major depression to controls and found no difference in AD pathology, but did find an increase in atheromatous disease in those with depression. O'Brien et al ${ }^{13}$ compared depressed participants with and without cognitive impairment and found no difference in AD pathology or microvascular disease. Clearly, more research needs to be done to determine the basis of the association between AD and depression.

During autopsy, AD pathology is sometimes found in people who are cognitively normal before death. Some hypothesize this represents presymptomatic AD, arguing that these people died before the onset of clinical symptoms. ${ }^{14}$ Alternatively, it is possible that these individuals are resilient and would have never developed dementia. The latter is supported by Schmitt et $\mathrm{al}^{15}$ who found that among cognitively normal people with AD pathology at autopsy, $61 \%$ also had microinfarcts and yet displayed no evidence of cognitive or functional decline.

Given the association between AD and depression in the preclinical phase of $\mathrm{AD}$, and the potential involvement of AD pathology in the development of late life depression, this autopsy study examined the rates of depression in cognitively normal individuals with AD pathology at autopsy. In line with the hypothesis that AD pathology leads to depression, it was hypothesized that cognitively normal individuals with AD pathology would have (1) higher rates of depression than cognitively normal individuals with no significant AD pathology, and (2) comparable rates of depression with those seen in individuals with $\mathrm{AD}$ pathology and mild cognitive impairment (MCI) or individuals with definite AD (dementia plus AD pathology).

\section{METHODS}

\section{Design}

A nested case-control study was conducted to compare rates of depression before death in 4 groups of individuals: (1) cognitively normal controls with no AD pathology, (2) cognitively normal with AD pathology, (3) MCI with AD pathology, and (4) dementia with AD pathology.

\section{Procedure}

Participant data were obtained from the Baltimore Longitudinal Study of Aging (BLSA) and consisted of a subset of participants in the BLSA who came to brain autopsy. The BLSA consists of a cohort of 1200 community-dwelling volunteer participants. Since 1986, 579 participants were followed by the National Institute on Aging in collaboration with the Johns Hopkins
Alzheimer Disease Research Center (JHADRC). ${ }^{16}$ Participants returned for visits approximately every 2 years and are examined with a battery of neuropsychologic tests.

All participants were reviewed at a consensus conference if their Blessed Information Memory Concentration score ${ }^{17}$ was $\geq 3$, if their informant or subject Clinical Dementia Rating $(\mathrm{CDR})^{18}$ score was $\geq 0.5$, or if the Dementia Questionnaire (DQ) ${ }^{19}$ was abnormal. At these conferences, participant information is presented to a consensus panel of experts consisting of neurologists and neuropsychologists that yields one of the following cognitive diagnoses: cognitively normal, MCI, or dementia. All participants, regardless of screening tests, were evaluated by case conference at the time of death or withdraw. Diagnoses of dementia were made at a consensus diagnostic conference using the Diagnostic and Statistical Manual of Mental Disorders, Third Edition, Revised (DSM-III-R) criteria ${ }^{20}$ and the National Institute of Neurological and Communication Disorders-Alzheimer's Disease and Related Disorders Association criteria. ${ }^{21}$ The full panel of neuropsychologic diagnostic tests and clinical data were available for review at the case conference. A diagnosis of MCI not meeting criteria for dementia was made for participants who had impairment in either a single cognitive domain (usually memory) or had more than one cognitive impairment but did not have functional loss in activities of daily living. Diagnostic findings most proximal to each participant's death were used to determine their cognitive diagnostic status for this study.

At enrollment, BLSA participants are offered the opportunity to participate in the brain autopsy component of the study. Those who agree are enrolled in an autopsy registry. Brain autopsy is performed by pathologists at Johns Hopkins with an average postmortem interval of 13 hours. The left hemibrain, used for morphologic studies, was fixed in $10 \%$ buffered formaldehyde for 2 weeks. Histologic examination of the brain included hippocampus, entorhinal cortex, middle frontal gyrus [Brodmann's area (BA) 46], middle and superior temporal gyri (BA 21 and 22), inferior parietal cortex (BA 39), and occipital cortex (BA 17 and 18). Sections (10- $\mu \mathrm{m}$ thick) were stained with hematoxylin-eosin and the Hirano modification of the Bielchowsky silver method ${ }^{22}$ and immunostained with antibodies against tau, ubiquitin, and $\alpha$-synuclein. Using silver-stained histologic sections, the presence and frequency of neuritic amyloid plaques was assessed following the Consortium to Establish a Registry for Alzheimer's Disease (CERAD) guidelines. $^{23}$ According to these criteria, each cortical region is examined at $100 \times$ magnification and the frequency of neuritic plaques is assessed as none, sparse, moderate, or frequent. Then, on the basis of the highest frequency on any single section, an age-related plaque score is assigned. This score could be 0 for those brains with no neuritic plaques and $\mathrm{A}, \mathrm{B}$, or $\mathrm{C}$ for those with sparse, moderate, or frequent neuritic plaques, respectively. The distribution of NFT was evaluated following the Braak scoring system. ${ }^{24}$ 
Brains were examined for vascular lesions gross and microscopically and included large infarcts (larger than $5 \mathrm{~mm}$ ), lacunes (5 $\mathrm{mm}$ or smaller), and microinfarcts (lesions detected microscopically but not on gross examination). Immunostains described above were used to assess for other neurodegenerative processes, that is, Lewy body pathology and tauopathies.

On the basis of consensus clinical diagnosis and the neuropathologic observations, the participants were divided into 4 postmortem groups: (1) Normal: subjects with normal cognition and no significant AD pathology (CERAD plaque score 0 or A; Braak NFT score $\leq 2$ ); (2) pathAD subjects with normal cognition and AD pathology (CERAD plaque score B or C; Braak NFT score $\geq 2$ ); (3) MCI: subjects with MCI and AD pathology (CERAD plaque score B or C; Braak NFT score $\geq 2$ ); and (4) AD: subjects with $\mathrm{AD}$ dementia and $\mathrm{AD}$ pathology (CERAD plaque score B or C; Braak NFT score $\geq 2$ ). All brains with significant cerebrovascular, $\alpha$-synuclein or tau lesions, or pathologic changes indicating other etiologies of dementia were excluded so that the association between AD pathology and depression could be examined independently of other potential confounding brain pathologies. Of the 168 participants that came to autopsy, 83 met the criteria to be selected and were included in the study.

\section{Measures}

Depressive symptoms were assessed using the Center for Epidemiologic Studies Depression Scale (CES-D). ${ }^{25}$ The CES-D is a 20-item inventory of depressive symptoms, and each response is scored from 0 to 3 based on the frequency of occurrence of the symptom. The range of scores is 0 to 60 , where higher CES-D scores indicate increased frequency and severity of depressive symptoms. A CES-D score of 16 or higher is suggestive of clinical depression. ${ }^{26}$ However, the CES-D does not make a diagnosis of clinical depression. It is a screening tool designed for general population surveys and for use in epidemiologic studies of the association between depression and other variables of interest. ${ }^{25}$ The CES-D is a highly reliable measure with an internal consistency of 0.85 in the general population, ${ }^{25}$ and it has been extensively validated in many different populations including elderly populations. ${ }^{26-28}$ Participants were administered the CES-D Scale at each visit before their death for an average of 4.11 (range, 1 to 12) times/visits.

\section{Analytic Plan}

Baseline CES-D scores, baseline age, age at death, time interval between first clinical visit and death, time interval between last clinical visit and death, number of visits and Braak and CERAD scores were first compared across groups (Normal, pathAD, MCI, and AD) as continuous variables using a 1-way analysis of variance. Means were compared by $t$ tests to examine the difference between specific groups when the analysis of variance values reached significance based on an a priori value of
$P<0.05$. CES-D scores were also analyzed as a categorical variable, dichotomized at the standard cutoff of $\geq 16$ to define clinically significant symptoms of depression. The proportion of individuals with clinically significant symptoms of depression at baseline and sex were compared across groups using Fischer exact test.

The longitudinal relationship between depression and Alzheimer pathology was examined using generalized estimating equations. ${ }^{29}$ Generalized estimating equation is advantageous over other longitudinal methods because it does not assume a normal distribution of the dependent variable, considers the within-person correlation when examining multiple observations per subject, does not require a balanced design, and can be used for both continuous and dichotomous outcomes. When examining the relationship between Alzheimer pathology and depressive symptoms using the CES-D as a continuous outcome, a gamma distribution, log link and exchangeable correlation matrix was incorporated. For analyses focusing on clinically significant depression using a dichotomous outcome (CES-D $\geq 16$ ), a binary distribution, probit link, and exchangeable correlation matrix was used and odds ratios (OR) were calculated. In all models, pathology group was coded as a dummy variable with pathAD as the reference category. Each model included terms for pathology group $\times$ time interaction. However, as no interactions were found to be significant at either the $P<0.05$ or $P<0.10$ level, these coefficients were not included in the final models. Age at death and time were controlled for in the analyses. All analyses were conducted using Stata Version 9.0 (StataCorp, College Station, TX).

\section{RESULTS}

The 4 groups did not differ in sex or age at first visit (Table 1), but did differ in age at death. Participants with pathologic $\mathrm{AD}$ only, MCI plus $\mathrm{AD}$ pathology, and dementia plus AD pathology all died significantly older and had longer time intervals between first visit and death compared with the cognitively normal controls. Participants with dementia also had a longer time interval from first visit to death than participants with pathologic $\mathrm{AD}$ only. The groups also differed in time between last visit and death (Table 1). Participants with dementia plus AD pathology had a longer time interval between the last clinical visit and death than normal controls and participants with pathologic AD only. Participants with pathologic AD only had more visits than either the normal or AD groups.

As expected, the groups differed with regard to brain pathology findings (Table 1). Mean Braak scores were significantly different between the groups. Participants with pathologic AD only, MCI, and participants with dementia all had higher Braak scores than cognitively normal controls. Participants with dementia also had significantly higher Braak scores than participants with pathologic AD only. CERAD scores were lower in 
TABLE 1. Characteristics of Study Population

\begin{tabular}{|c|c|c|c|c|c|}
\hline & Normal $(N=24)$ & PathAD $(\mathrm{N}=20)$ & $\operatorname{MCI}(N=13)$ & $\operatorname{AD}(\mathbf{N}=26)$ & Total $(\mathbf{N}=\mathbf{8 3})$ \\
\hline Male (n, \%) & $20(87.0 \%)$ & $14(70.0 \%)$ & $10(76.9 \%)$ & $16(61.5 \%)$ & $60(73.2 \%)$ \\
\hline \multicolumn{6}{|l|}{ Age at visit 1} \\
\hline Mean (SE) & $73.3(1.9)$ & $77.0(1.4)$ & $78.1(1.8)$ & $77.6(1.5)$ & $76.3(0.9)$ \\
\hline \multicolumn{6}{|l|}{ Age at death } \\
\hline \multicolumn{6}{|l|}{ Time interval (y) visit 1 to death } \\
\hline Mean (SE) & $7.2(0.7)$ & $10.4(0.8)^{* \dagger}$ & $11.0(1.2)^{*}$ & $13.0(0.8)^{*}$ & $10.4(0.5)$ \\
\hline \multicolumn{5}{|l|}{ Time interval (y) last visit to death } & $3.6(0.4)$ \\
\hline Mean (SE) & $3.0(0.4)$ & $5.8(0.7)^{*} \dagger$ & $4.4(0.8)$ & $3.7(0.4)$ & $4.1(0.3)$ \\
\hline \multicolumn{6}{|l|}{ Braak score } \\
\hline Mean (SE) & $2.0(0.1)$ & $3.1(0.3)^{*} \dagger$ & $3.3(0.3)^{*}$ & $4.2(0.3)^{*}$ & $3.1(0.2)$ \\
\hline \multicolumn{6}{|l|}{ CERAD score } \\
\hline Mean (SE) & $0.1(0.1)$ & $1.8(0.2)^{*}$ & $1.69(0.3)^{*}$ & $2.1(0.2)^{*}$ & $1.4(0.1)$ \\
\hline
\end{tabular}

*Significantly different from normal control group $(P<0.05)$.

$\dagger$ Significantly different from AD group $(P<0.05)$.

AD indicates dementia with Alzheimer pathology; CERAD, Consortium to Establish a Registry for Alzheimer's Disease [scores: $0=$ none; $1=$ rare $(A) ; 2=$ moderate (B); 3 = frequent (C)]; MCI, MCI with Alzheimer pathology; Normal, cognitively and pathologically normal controls; pathAD, cognitively normal with Alzheimer pathology.

the normal group and did not significantly differ between the 3 pathology groups.

\section{Depression}

At baseline, there were no differences between groups in number of depressive symptoms or percent of participants with CES-D $\geq 16$ (data not shown). The longitudinal association between pathology group and CES-D, as a continuous scale, was then examined controlling for age at death and time. Compared with the pathAD group, the AD $(b=0.436, \mathrm{SE}=0.18$, $P=0.016)$ and cognitively normal $(b=0.351, \mathrm{SE}=0.20$, $P=0.074)$ groups, on average, had higher levels of depressive symptoms over the course of the follow-up (Table 2). There were no group $\times$ time interactions. The findings between AD pathology and depressive symptoms extended to clinically significant depression. Compared with the pathAD group, the cognitively normal $(\mathrm{OR}=1.61, P=0.050)$ and $\mathrm{AD}$ groups $(\mathrm{OR}=1.57$, $P=0.045)$ had higher odds of depression $(\mathrm{CES}-\mathrm{D} \geq 16$ ) (Table 2). Again, there were no group $\times$ time interactions.

TABLE 2. Longitudinal Association Between CES-D (Continuous and Dichotomous) and Alzheimer Pathology Groups*

\begin{tabular}{lcccc}
\hline Group & $\begin{array}{c}\text { CES-D as a Continuous Variable } \\
\text { ( } \beta \text {-Coefficient) }\end{array}$ & $\boldsymbol{P}$ & $\begin{array}{c}\text { CES-D } \\
\geq \mathbf{1 6}(\text { OR) }\end{array}$ & $\boldsymbol{P}$ \\
\hline PathAD & Ref & & Ref & \\
Normal & 0.351 & 0.074 & 1.61 & 0.050 \\
MCI & 0.113 & 0.596 & 1.13 & 0.204 \\
AD & 0.436 & 0.016 & 1.57 & 0.045
\end{tabular}

*Controlling for age at death and time.

AD indicates dementia with Alzheimer pathology; MCI, MCI with Alzheimer pathology; Normal, cognitively and pathologically normal controls; pathAD, cognitively normal with Alzheimer pathology.

\section{DISCUSSION}

This autopsy study examined rates of depression in individuals with pathologic $\mathrm{AD}$ but no cognitive decline before death. It was predicted that these individuals would have higher rates of depression than cognitively and pathologically normal controls given the hypothesis that pathologic AD leads to depression. Surprisingly, individuals with pathologic AD only had lower rates of depression and less depressive symptoms when compared with normal controls and to participants with dementia plus AD pathology. Together, these data suggest that AD pathology alone is not associated with depression. Furthermore, these data suggest that the individuals with pathologic AD only appear to be resilient to the development of brain symptoms, as they had neither depression nor cognitive decline despite having significant AD pathology. Taken together, these findings support the association between depression and dementia of AD, but suggest that this association is independent of $\mathrm{AD}$ pathology, as cognitively normal individuals with pathologic AD had lower rates of depression. This association occurred in the absence of significant brain vascular pathology, as participants with vascular pathology at autopsy were excluded.

Previous studies have suggested that those with pathologic AD but no cognitive decline before death represent presymptomatic AD individuals who died before the onset of clinical symptoms. ${ }^{14}$ Our findings suggest that these individuals are resilient to brain symptoms and may never have developed dementia. In addition, previous studies have suggested that depression is either a risk factor for dementia ${ }^{4-6}$ or a preclinical state of $\mathrm{AD} .^{7-9}$ The role of $\mathrm{AD}$ pathology in depression is unclear as some studies have suggested it is involved, ${ }^{10}$ whereas others have shown it is not involved. ${ }^{1-13}$ The results of the current study suggest that depression is a risk factor for $\mathrm{AD}$ in the presence of $\mathrm{AD}$ pathology, but 
depression is not a risk factor for AD pathology. Thus, another factor may underlie the association between depression and AD.

Several hypotheses have been proposed to explain the association between depression and AD. It has been suggested that depression in $\mathrm{AD}$ may be due to a preferential loss of neurons in the locus ceruleus, ${ }^{30}$ but more recent studies have not found an increased loss of neurons in individuals with AD and depression compared to those with AD without depression. ${ }^{31}$ Similarly, few differences have been found in the loss of serotonergic neurons in the dorsal raphe nuclei between AD patients with and without depression. ${ }^{32}$ However, AD pathology may prompt genetically vulnerable people to have more neuronal loss in these areas leading to depression. Holmes et $\mathrm{al}^{33}$ found that individuals with $\mathrm{AD}$ who had certain polymorphisms in the serotonin receptor genes 5-HT2A and 5-HT2C were more likely to be depressed. In addition, Sheline et $\mathrm{a}^{34}$ found smaller hippocampal volumes in individuals with a history of major depression and suggested this may be a result of glucocorticoid-induced neurotoxicity. However, this etiologic mechanism remains controversial as most of the supporting evidence is indirect ${ }^{35}$ and more recent studies have not shown an association between hypercortisolemia and hippocampal volumes. ${ }^{36}$ Lopez et $\mathrm{al}^{37}$ recently found higher rates of depression in patients with AD who had Lewy bodies in the amygdala, suggesting that comorbid Lewy body pathology may be associated with depression in AD. Finally, it is also possible that depression may accelerate existing $\mathrm{AD}$ pathology leading to dementia.

The strengths of this study include that it was autopsy based, such that we were able to separate out AD pathology from other brain pathologies. In addition, by using the BLSA data, we had multiple CES-D scores over a long follow-up period of up to 24 years. This study also has several limitations. The BLSA participants are a select population that volunteered to participate in a life-long project. These individuals have higher levels of education and socioeconomic status than the general population. Therefore, our findings may not be representative of the general population. Another reason that our findings may not be generalizable is that $73 \%$ of participants were male. This sex disproportion may be important considering the fact that the prevalence of LLMD is twice as high in females compared with men. ${ }^{38}$ Another limitation of this study is the measure of depression. Although the CES-D is a widely used and validated screening tool, it does not make a clinical diagnosis of depression. Furthermore, the diagnosis of depression in AD can be difficult as symptoms of other neuropsychiatric manifestations of $\mathrm{AD}$, such as apathy, often overlap with symptoms of depression. ${ }^{39}$ The testers in this study are sensitive to motivational issues, particularly in participants who feel they are declining. They use a variety of techniques to encourage people to do the best they can regardless of how they feel at the moment. However, a more ideal measure of depression would be a structured clinical assessment performed by an experienced geriatric psychiatrist.

Also of note, the cognitively normal controls without AD pathology died significantly younger than the other 3 groups with AD pathology. We would expect the opposite to be true, that the groups with $\mathrm{AD}$ pathology would die younger because AD pathology accelerates death. It is not clear why the normal controls died significantly younger or what effect, if any, this difference has on our results. It is possible that the normal controls died younger than the groups with AD pathology owing to other medical illnesses that have been associated with comorbid depression such as coronary heart disease or cancer, ${ }^{40}$ which may have affected the level of depressive symptoms in the normal control group. However, CES-D scores did not increase before death in the normal control group, as might be expected if the depressive symptoms were related to comorbid disease.

Future studies should replicate these findings with refined hypotheses. Amyloid imaging may be a useful modality to replicate this study in living participants. If it is confirmed that the association between depression and $\mathrm{AD}$ is independent of AD pathology, then future studies should seek to identify the underlying basis of this association. Furthermore, if the cognitively normal individuals with AD pathology in our study are truly resilient to brain symptoms, future studies that would better characterize these individuals may have implications for prevention of symptoms of depression and dementia.

\section{REFERENCES}

1. Hebert LE, Scherr PA, Bienias JL, et al. Alzheimer disease in the U.S. population: prevalence estimates using the 2000 census. Arch Neurol. 2003;60:1119-1122.

2. Lyketsos CG, Olin J. Depression in Alzheimer's disease: overview and treatment. Biol Psychiatry. 2002;52:243-252.

3. Lyketsos CG, Steinburg M, Tschanz JT, et al. Mental and behavioral disturbances in dementia: findings from the Cache County study on memory in aging. Am J Psychiatry. 2000;157: 708-714

4. Wilson RS, Barnes LL, Mendes de Leon CF, et al. Depressive symptoms, cognitive decline, and risk of $\mathrm{AD}$ in older persons. Neurology. 2002;59:364-370.

5. Dal Forno G, Palermo MT, Donohue JE, et al. Depressive symptoms, sex, and risk for Alzheimer's disease. Ann Neurol. 2005;57:381-387.

6. Cannon-Spoor HE, Levy JA, Zubenko GS, et al. Effects of previous major depressive illness on cognition in Alzheimer disease patients. Am J Geriatr Psychiatry. 2005;13:312-318.

7. Berger AK, Fratiglioni L, Forsell Y, et al. The occurrence of depressive symptoms in the preclinical phase of AD: a populationbased study. Neurology. 1999;53:1998-2002.

8. Geerlings MI, Schoevers RA, Beekman ATF, et al. Depression and risk of cognitive decline and Alzheimer's disease: results of two prospective community-based studies in The Netherlands. Br J Psychiatry. 2000;176:568-575.

9. Gatz JL, Tyas SL, St. John P, et al. Do depressive symptoms predict Alzheimer's disease and dementia? J Gerontol. 2005;60:744-747.

10. Sweet RA, Hamilton RL, Butters MA, et al. Neuropathologic correlates of late-onset major depression. Neuropsychopharmacology. 2004;29:2242-2250. 
11. Wilson RS, Schneider JA, Bienias JL, et al. Depressive symptoms, clinical $\mathrm{AD}$, and cortical plaques and tangles in older persons. Neurology. 2003;61:1007-1102.

12. Thomas AJ, Ferrier IN, Kalaria RN, et al. A neuropathological study of vascular factors in late-life depression. J Neurol Neurosurg Psychiatry. 2001;70:83-87.

13. O'Brien J, Thomas A, Ballard C, et al. Cognitive impairment in depression is not associated with neuropathologic evidence of increased vascular or Alzheimer-type pathology. Biol Psychiatry. 2001;49:130-136.

14. Morris JC, Storandt M, McKeel DW Jr. Cerebral amyloid deposition and diffuse plaques in "normal" aging: evidence for presymptomatic and very mild Alzheimer's disease. Neurology. 1996;46:707-719.

15. Schmitt FA, Davis DG, Wekstein DR, et al. "Preclinical" AD revisited: neuropathology of cognitively normal older adults. Neurology. 2000;55:370-376.

16. Kawas C, Gray S, Brookmeyer R, et al. Age-specific incidence rates of Alzheimer's disease: the baltimore longitudinal study of aging. Neurology. 2000;54:2072-2077.

17. Blessed G, Tomlinson BE, Roth $\mathrm{M}$. The association between quantitative measures of dementia and of senile change in the cerebral gray matter of elderly subjects. Br J Psychiatry. 1968;114: 797-811.

18. Morris JC.. Clinical dementia rating: a reliable and valid diagnostic and staging measure for dementia of the Alzheimer type. Int Psychogeriatr. 1997:9(suppl 1):173-178.

19. Kawas C, Segal J, Stewart WF, et al. A validation study of the Dementia Questionnaire. Arch Neurol. 1994;51:901-906.

20. American Psychiatric Association. Diagnostic and Statistical Manual of Mental Disorders, 3rd edition, revised. Washington, DC: American Psychiatric Association; 1987.

21. McKhann G, Drachman D, Folstein M, et al. Clinical diagnosis of Alzheimer's disease: report of the NINCDS-ADRDA Work Group under the auspices of Department of Health and Human Services Task Force on Alzheimer's disease. Neurology. 1984;34:939-944.

22. Yamamoto T, Hirano A. A comparative study of modified Bielschowsky, Bodian and thioflavin S stains on Alzheimer's neurofibrillary tangles. Neuropathol Appl Neurobiol. 1986;12:3-9.

23. Mirra SS, Heyman A, McKeel D, et al. The Consortium to Establish a Registry for Alzheimer's Disease (CERAD). Part II. Standardization of the neuropathologic assessment of Alzheimer's disease. Neurology. 1991;41:479-486.

24. Braak H, Braak E. Neuropathological staging of Alzheimer-related changes. Acta Neuropathol. 1991;82:239-259.
25. Radloff LS. The CES-D Scale: a self-report depression scale for research in the general population. Appl Psychol Measure. 1977;1: 385-401.

26. Weissman MM, Sholomskas D, Pottenger M. Assessing depressive symptoms in five psychiatric populations: a validation study. $\mathrm{Am} \mathrm{J}$ Epidemiol. 1977;106:203-214.

27. Beekman AT, Deeg DJ, Van Limbeek J, et al. Criterion validity of the Center for Epidemiologic Studies Depression scale (CES-D): results from a community-based sample of older subjects in The Netherlands. Psychol Med. 1997;27:231-235.

28. Davidson H, Feldman PH, Crawford S. Measuring depressive symptoms in the frail elderly. J Gerontol. 1994;49:159-164.

29. Zeger SL, Liang KY. Longitudinal data analysis for discrete and continuous outcomes. Biometrics. 1986;42:121-130.

30. Forstl H, Burns A, Luthert P, et al. Clinical and neuropathological correlates of depression in Alzheimer's disease. Psychol Med. 1992;22:877-884.

31. Hoogendijk WJG, Sommer IEC, Pool CW, et al. Lack of association between depression and loss of neurons in the locus coeruleus in Alzheimer disease. Arch Gen Psychiatry. 1999;56: $45-51$.

32. Hendricksen M, Thomas AJ, Ferrier IN, et al. Neuropathological study of the dorsal raphe nuclei in late-life depression and Alzheimer's disease with and without depression. Am J Psychiatry. 2004;161:1096-1102.

33. Holmes C, Arranz M, Collier D, et al. Depression in Alzheimer's disease: the effect of serotonin receptor gene variation. Am J Med Genetics Part B Neuropsychiatr Genetics. 2003;119B:40-43.

34. Sheline YI, Wang PW, Gado MH, et al. Hippocampal atrophy in recurrent major depression. Proc Natl Acad Sci. 1996;93:3908-3913.

35. Sapolsky RM. Glucocorticoids and hippocampal atrophy in neuropsychiatric disorders. Arch Gen Psychiatry. 2000;57:925-935.

36. O'Brien JT, Lloyd A, McKeith I, et al. A longitudinal study of hippocampal volume, cortisol levels, and cognition in older depressed subjects. Am J Psychiatry. 2004;161:2081-2090.

37. Lopez OL, Becker JT, Sweet RA, et al. Lewy bodies in the amygdala increase risk for major depression in subjects with Alzheimer disease. Neurology. 2006;67:660-665.

38. Sonnenberg CM, Beekman ATF, Deeg DJH, et al. Sex differences in late-life depression. Acta Psychiatr Scand. 2000;101:286-292.

39. Lee HB, Lyketsos CG. Depression in Alzheimer's Disease: heterogeneity and related issues. Biol Psychiatry. 2003;54:353-362.

40. Krishnan KR, Delong M, Kraemer $\mathrm{H}$, et al. Comorbidity of depression with other medical diseases in the elderly. Biol Psychiatry. 2002;52:559-588. 